\title{
Editorial: Theories and Applications in Network Science
}

\author{
Roy Cerqueti ${ }^{1,2}$ and Feng Shi ${ }^{3,4 *}$ \\ ${ }^{1}$ Sapienza University Rome, Rome, Italy, ${ }^{2}$ London South Bank University, London, United Kingdom, ${ }^{3}$ TigerGraph, Redwood \\ City, CA, United States, ${ }^{4}$ Amazon, Seattle, WA, United States
}

Keywords: complex networks, network science, community detection, neural networks, social networks

\section{Editorial on the Research Topic}

\section{Theories and Applications in Network Science}

With a few decades of development, networks have become a go-to mathematical model for analyzing various complex systems in the real world, whether physical, biological, economic, or social. Researchers from diverse fields have developed a wide range of techniques to address problems in such networks, integrating knowledge from math, physics, computer science, and beyond.

In general, networks are mathematical models that describe unified frameworks composed of disaggregated components-the nodes-by including components' interconnections. Network models are then a step beyond the simple case of systems collecting different unrelated entities; indeed, the links between the nodes provide unique information on the investigated phenomena.

The polyhedric nature of networks can be appreciated when exploring the related methodological contributions and their applications. In this respect, we first mention community detection in a network, the identification of special subsets of nodes that are densely connected within each subset but sparsely connected between subsets. Community detection contexts can be found also in one paper published in the Research Topic. Indeed, this method is used by Turnbull and O'Neale in the study of education networks. Along the lines of forming connections between nodes, another active area of research is the formation of links in the light of stochastic evolution of networks and the assessment of the probability that two disconnected nodes will form a link.

Centrality measures is another big methodological topic, and they are commonly used to extract important features of the related networks in practice. Popular use cases include ranking nodes in a network, finding relationships between links and nodes' attributes and network topology, and identifying hubs or certain crucial nodes whose removal would disconnect the system. Two contributions in the Research Topic- Bellingeri et al. and Schweitzer et al. - are interesting applications in this area.

Undoubtedly, network science is way broader than the short introduction above. Considering node and edge types, edge weights and directions, temporal features, and their combinations, there are so many ways one can model a network system, which makes the field both challenging and exciting.

The contributions in the present Research Topic aim to present a high-quality panoramic view

Received: 24 November 2021 Accepted: 03 December 2021 Published: 22 December 2021 of the scientific debate at the frontier of network science, as briefly discussed below.

Bellingeri et al. present a complex networks-based epidemic model in the framework of the COVID-19 spread. The authors elaborate on the connection between the removal of social links and diffusion of the pandemics in the light of non-pharmaceutical interventions.

Schweitzer et al. are somehow in the line of Bellingeri et al. by exploring the resilience of a network in the case of fluctuations due to nodes removal. They deal with the particular case of collaboration networks in the context of R\&D when the nodes are innovating firms and the 
links capture the presence of interconnections among them. Interestingly, the authors show that the network structure can mitigate the detrimental effects of the firms leaving the collaboration structure in a relevant empirical instance.

Gao and Fan build a network model in the paradigmatic environment of systemic risk. The authors present the empirical case of a large set of Chinese banks by discussing the impact on them of an external shock; then, they elaborate on the construction of optimal portfolio strategies for banks' optimal investments.

On neural networks in the brain, Kwessi studies a mathematical model and Wang et al. present an interesting application of network science-identify potential brain regions related to Alzheimer's disease (AD).

Kwessi proposes a discrete model for dynamical neural fields and proof of the existence of its non-trivial solutions. Illustrating with simulations, the author also shows and discusses the stability of the solutions. The author finds quite a few interesting mathematical properties of the system, which may contribute to the theoretical understanding of how neurons in the brain work.

Wang et al. propose a new index derived from four common network features and a novel 2-hopconnectivity, also proposed by the authors in this work. With literature verification and canonical correlation analysis, the new index is shown to capture certain structural properties of the neural networks of $\mathrm{AD}$ patients and might be a promising tool for detecting and tracking disease progression.

Turnbull and O'Neale analyze a co-enrollment network of high school students in Aotearoa New Zealand, where nodes are "standards" (analogous to subjects or topics but not the same) and edges correspond to how often two standards are chosen by same students. They find network structures differ between subpopulations based on students' gender, ethnicity, and social-economic status. Their findings shed new light on STEM education in New Zealand and they provide tools that might help promote more fair and effective education in general.

Last but not least, Ogle et al. demonstrate how to use a networking framework-Named Data Networking, an experiment architecture for distributed internet-to manage the explosion of genomic data in biology. They also provide a prototype integrating NDN with a contemporary genomics workflow (GEMmaker) and illustrate the possibility of their proposal.

\section{AUTHOR CONTRIBUTIONS}

All authors listed have made a substantial, direct, and intellectual contribution to the work and approved it for publication.

Conflict of Interest: FS was employed by company TigerGraph and Amazon.

The remaining author declares that the research was conducted in the absence of any commercial or financial relationships that could be construed as a potential conflict of interest.

Publisher's Note: All claims expressed in this article are solely those of the authors and do not necessarily represent those of their affiliated organizations, or those of the publisher, the editors and the reviewers. Any product that may be evaluated in this article, or claim that may be made by its manufacturer, is not guaranteed or endorsed by the publisher.

Copyright (c) 2021 Cerqueti and Shi. This is an open-access article distributed under the terms of the Creative Commons Attribution License (CC BY). The use, distribution or reproduction in other forums is permitted, provided the original author(s) and the copyright owner(s) are credited and that the original publication in this journal is cited, in accordance with accepted academic practice. No use, distribution or reproduction is permitted which does not comply with these terms. 